\title{
Qu Feng Xuan Bi Formula Alleviates Airway Inflammation and Guides Clinical Treatment by Improving Bronchial Immune Balance in the Asthma Model
}

\author{
Po Hu, ${ }^{1}$ Suofang Shi $\mathbb{D}^{2}{ }^{2}$ Li Liu, ${ }^{3}$ Shuomiao Yin, ${ }^{4}$ and Lingfu Wang' \\ ${ }^{1}$ The First Clinical Medical College, Nanjing University of Traditional Chinese Medicine, Nanjing, Jiangsu 210029, China \\ ${ }^{2}$ Department of Respiratory Diseases, The First Affiliation Hospital of Nanjing University of Traditional Chinese Medicine, \\ Nanjing, Jiangsu 210029, China \\ ${ }^{3}$ Department of Molecular Biology, The First Affliation Hospital of Nanjing University of Traditional Chinese Medicine, Nanjing, \\ Jiangsu 210029, China \\ ${ }^{4}$ The First Clinical Medical College, Guangzhou University of Traditional Chinese Medicine, Guangzhou, \\ Guangdong 510006, China
}

Correspondence should be addressed to Suofang Shi; zqgxc1234@163.com

Received 23 November 2020; Revised 6 December 2020; Accepted 8 January 2021; Published 21 January 2021

Academic Editor: Qinhu Zhang

Copyright $(2021$ Po Hu et al. This is an open access article distributed under the Creative Commons Attribution License, which permits unrestricted use, distribution, and reproduction in any medium, provided the original work is properly cited.

\begin{abstract}
Network pharmacology analysis has shown that Qu Feng Xuan Bi Formula (QFXBF) is a traditional Chinese herbal medicine used to treat bronchial asthma, with demonstrated remarkable effects; however, its effects on airway inflammation and bronchial asthma alleviation remain unclear. This study aimed to explore the underlying mechanism of QFXBF by assessing toll-like receptor-9 (TLR-9) and the downstream MAPK signaling pathway as well as the immune modulation of the Th1/Th2 balance. The results showed that the aqueous extract of QFXBF increased TLR-9 expression, regulated the downstream genes AP-1, JNK, and ERK1/2, and reduced serum IL-5 levels in the high-dose QFXBF group; meanwhile, following QFXBF treatment, IFN- $\gamma$ and TNF$\alpha$ levels were increased, indicating that the Th1/Th2 immune balance was reversed as predicted. These findings provide a mechanism underlying QFXBF's effects, which significantly improves the treatment and management of patients with bronchial asthma and profoundly impacts medical information processing.
\end{abstract}

\section{Introduction}

Bronchial asthma, a chronic disease characterized by eosinophilic airway inflammation, goblet cell metaplasia, and bronchial hyperreactivity (BHR), is controlled by innate and adaptive immune responses to inhaled allergens via pattern recognition receptors (PRRs) on barrier epithelial cells (ECs) and dendritic cells (DCs) [1]. It is a highly prevalent chronic lung condition affecting more than 300 million people worldwide, with up to 250,000 deaths per year and billions of health care costs [2]. Although regular use of inhaled corticosteroids (ICSs) effectively controls asthma symptoms and reduces the mortality rate, asthma remains a clinical challenge with an increasing incidence of related attacks. Except for glucocorticoids, leukotriene receptor antagonists, $\beta$-agonists, and other available therapeutic drugs, novel drugs, including TLR-9 agonists, are still at an early stage of development [3]. Despite previous findings that these drugs may be useful, there is insufficient evidence to support their asthma application, and their exact effects remain unknown [4]. New medications that can overcome the existing deficiencies in traditional asthma drugs are urgently needed [5].

In Jiangsu Provincial Hospital of Traditional Chinese Medicine (China), QFXBF has been used for 20 years to treat bronchial asthma according to traditional Chinese medicine principles strictly. Network pharmacology analysis showed that Qu Feng Xuan Bi Formula (QFXBF) is a traditional Chinese herbal medicine used to treat bronchial asthma and has shown remarkable effects. Our 
previous clinical observations have shown that the QFXBF significantly reduces acute exacerbation of asthma symptoms, reduces acute and chronic airway inflammation, and delays airway remodeling.

In a previous experimental study, we demonstrated that QFXBF significantly reduces airway hyper-responsiveness in a histamine-induced rat asthma model and decreases the amounts of eosinophils in the bronchoalveolar lavage fluid; however, the specific underlying mechanism is largely unknown. Previous findings suggested that serum levels of T-cell derived cytokines IL-13 and IL-4 decreased after high QFXBF treatment, while no overt changes were found in BALF samples [6].

TLR-9 activation suppresses Th2 immune responses in the allergic immune system to reduce eosinophilic infiltration in the lung and airway remodeling. In recent years, clinical studies assessing the activation of TLR-9 have focused on allergy (e.g., allergic rhinitis and allergic asthma), cancer, and infectious diseases [7]. AP-1 is a key nucleoprotein dimer downstream of the JNK and ERK pathways, consisting of c-Jun and c-Fos, and is involved in regulating the Th2-type cell differentiation and the regulation of cytokines such as IL-4, IL-5, and IL-13 [8]. Activation of AP-1 is one of the most important mechanisms by which lymphocytes are involved in chronic airway inflammatory responses. Specifically, external stimulation related to asthma, such as viruses and allergens, can activate intracellular AP-1 through a complex signaling pathway. Activated AP-1, as a nuclear messenger, could then turn the temporary stimulation activation signal on the cell surface into a signal for long-term gene transcription that enhances target genes for cell inflammation, resulting in increased expression of inflammatory proteins and maintaining chronic inflammation as shown in Figure 1 [9].

There are two major TLR-9 signaling pathways $[10,11]$, including the MAPK and NF- $\kappa$ B pathways. Both can cause inflammation of affect airway, but the respective downstream key factors and cytokines are different. The former eventually activates the p38, JNK, ERK1/2, and MAPK pathways, resulting in a subsequent increase in the level and release of cytokines such as the nuclear protein AP- 1 , ultimately affecting the nuclear regulation of multiple genes, inducing IL-4, IL-10, IL-13, and other inflammatory cytokines.

A recent study assessing specific T-cell subsets for cytokine gene expression showed that differentiation of Th1/ Th2 cells from Th0 cells could be divided into two main steps $[12,13]$. First, the chromosome structure in T-cells under antigen stimulation which brings the specific Th2 transcription factor GATA-3 closer to the transcription DNA sequence is rearranged. Secondly, other gene transcription factors, such as AP-1, bind these tissue-specific transcription factors and induce Th2 cells. Th2 cells, in turn, secrete IL- 4 , IL-5 and other cytokines, promote the synthesis of many IgE, and enhance the immune response that is closely related to asthma. ERK participates in asthma pathogenesis by affecting inflammation-mediated smooth muscle cell proliferation of the airway and remodeling the airway [14]. Indeed, the ERK signaling pathway is widely distributed in the airway smooth muscle; when the signaling pathway is activated, it accelerates the cell cycle, promotes DNA replication, increases the proliferation of airway epithelial cells, fibroblasts, and smooth muscle cells, and regulates inflammatory cytokines and other genes, ultimately leading to airway inflammation and remodeling [15].

This study aimed to assess key cytokines in the TLR-9 signaling pathways and determine whether phosphorylated proteins of the JNK and ERK pathways, downstream of MAPK signaling, e.g., AP-1 and ERK1/2, play a role in the treatment of asthma with QFXBF. The AP-1 dimer, composed of the c-Fos and c-Jun proteins, can be used as a nuclear messenger with temporary stimulus signals from the cell surface after activation and confers long-term gene transcription, which increases the frequency of transcription of target genes, upregulating inflammatory proteins and maintaining chronic inflammation. ERK1/2 was shown to promote epithelial cell proliferation by transducing an intracellular signal; in addition, it produces growth factors, including the TGF- $\beta$ family, fibroblast growth factor, and PDGF, which promote the transformation of lung fibroblasts into myofibroblasts, smooth muscle proliferation, and collagen deposition, finally causing airway remodeling.

\section{Materials and Methods}

2.1. Animals. Sixty healthy male guinea pigs (8-10 weeks; 200-240 g) were provided by the experimental animal center of Nantong University (experimental animal production license no. SCXK 2008-0010). Guinea pigs were free from pathogens and respiratory diseases and were used according to the guidelines of the All-University Committee on Animal Use and Care at Nantong Medical University. Experimental animals were kept in groups of two animals per cage. These cages are polycarbonate boxes covered with filtering covers, allowing free access to tap water and food chow. Animals were maintained at $12 \mathrm{~h}-12 \mathrm{~h}$ light $/$ dark cycle, $21-24^{\circ} \mathrm{C}$ room temperature, and $40-55 \%$ relative humidity. After adaptation, the animals were randomly divided into six experimental groups of 10 animals, including the (1) control (no treatment), (2) OVA only (animals treated with OVA and $\left.\mathrm{Al}(\mathrm{OH})_{3}\right)$, (3) DXM, (4) high-dose QFXBF, (5) medium dose QFXBF, and (6) low-dose QFXBF groups.

2.2. Extraction Protocol. The composition of QFXBF is shown in Table 1. The water-soluble extract was prepared as follows: $1,000 \mathrm{ml}$ of water was added to the plant material $(106 \mathrm{~g})$ and boiled for 45 minutes. After collection of the solution, add $500 \mathrm{ml}$ of water to the residual liquid and boil for further $45 \mathrm{~min}$, followed by the collection of fluid. The two extracts were filtered, concentrated, and finally packed. The final product contained $0.58 \mathrm{~g}$ of the original medicinal substance per $\mathrm{ml}$.

2.3. Chromatographic Analysis of QFXBF Sample. The extracted samples were stored at $-20^{\circ} \mathrm{C}$, with a shelf life of 18 months and stable quality. The four active ingredients in this prescription, tectorigenin $\left(\mathrm{C}_{16} \mathrm{H}_{12} \mathrm{O}_{6}\right)$, glycyrrhizic acid 


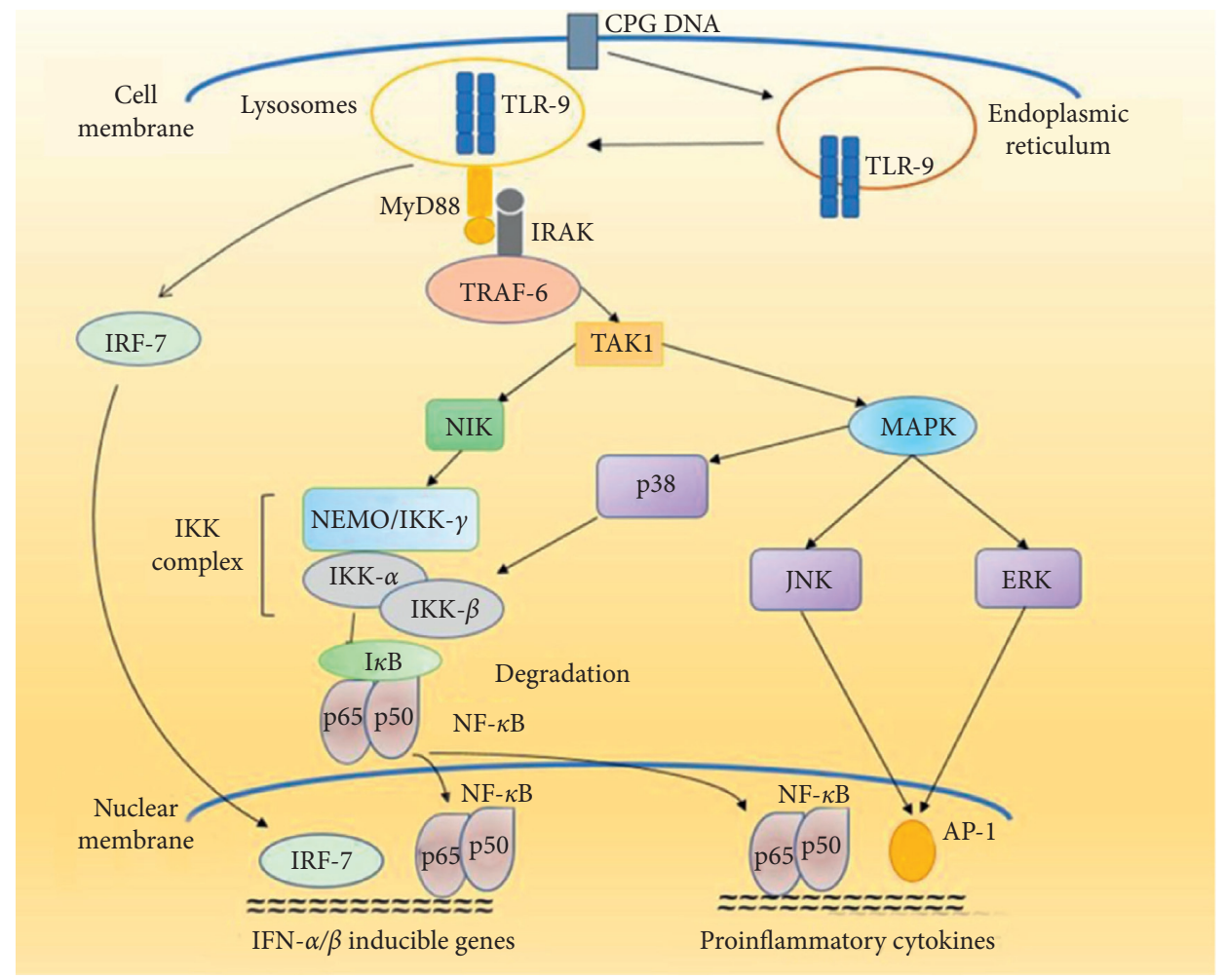

FIGURE 1: TLR9 signaling.

TABle 1: Composition of QFXBF.

\begin{tabular}{|c|c|c|c|c|c|}
\hline Herbal medicine & Scientific name & Family & Origin & Weight $(\mathrm{g})$ & Ratio (\%) \\
\hline Belamcandae Rhizoma & Belamcanda chinensis (L.) Redouté & Iridaceae & China & 10 & 10.0 \\
\hline Ephedrae Herba & Ephedra sinica Stapf & Ephedraceae & China & 5 & 5.0 \\
\hline Armeniacae Semen Amarum & Prunus armeniaca L. var. ansu Maxim & Rosaceae & China & 10 & 10.0 \\
\hline Glycyrrhizae Radix et Rhizoma & Glycyrrhiza uralensis Fisch & Leguminosae & China & 5 & 5.0 \\
\hline Pheretima & Pheretima aspergillum (E. Perrier) & Megascolecidae & China & 10 & 10.0 \\
\hline Trichosanthis Fructus & Trichosanthes kirilowii Maxim & Cucurbitaceae & China & 10 & 10.0 \\
\hline Allium macrostemon Bunge & Allium macrostemon Bunge & Liliaceae & China & 10 & 10.0 \\
\hline Bombyx Batryticatus & Bombyx mori Linnaeus & Bombycidae & China & 10 & 10.0 \\
\hline Kadsura Pepper Stem & Piper kadsura (Choisy) Ohwi & Piperaceae & China & 15 & 15.0 \\
\hline Cnidii Fructus & Cnidium monnieri (L.) Cuss & Umbelliferae & China & 15 & 15.0 \\
\hline Total amount & & & China & 100 & 100 \\
\hline
\end{tabular}

$\left(\mathrm{C}_{42} \mathrm{H}_{62} \mathrm{O}_{16}\right)$, amygdalin $\left(\mathrm{C}_{20} \mathrm{H}_{27} \mathrm{NO}_{11}\right)$, and osthole $\left(\mathrm{C}_{15} \mathrm{H}_{16} \mathrm{O}_{3}\right)$, were selected as the quantitative indicators, and their content in the final QFXBF product was analyzed by Agilent 1290 High-Performance Liquid Chromatograph using AB Triple TOF 5600 Quadrupole-Time of Flight Mass Spectrometer with Electrospray Ion Source (ESI), Analyst ${ }^{\circledR}$ TF 1.6 Workstation. The operating conditions were as follows: ZORBAX SB-C18 column $(2.1 \mathrm{~mm} \times 150 \mathrm{~mm}, 5 \mu \mathrm{m}$, Agilent), mobile phase: $0.1 \%$ aqueous formic acid (A)-acetonitrile (B), gradient elution (Table 2, Figure 2), flow rate: $200 \mu \mathrm{L} / \mathrm{min}$, column temperature: $40^{\circ} \mathrm{C}$, and injection volume: $10 \mu \mathrm{L}$.

2.4. Bronchial Asthma Modeling. The bronchial asthma model was established using ovalbumin (OVA) and $\mathrm{Al}(\mathrm{OH})^{3}$ gel sensitization. Animals were infused with OVA
TABLE 2: The gradient elution program of chromatographic analysis of the QFXBF sample.

\begin{tabular}{lcc}
\hline Time $(\mathrm{min})$ & A (\%) & B (\%) \\
\hline 0 & 95 & 5 \\
2 & 95 & 5 \\
2.5 & 70 & 30 \\
10 & 35 & 65 \\
10.5 & 5 & 95 \\
13 & 5 & 95 \\
15 & 95 & 5 \\
\hline
\end{tabular}

Ion source: ESI. Ion spray voltage/IS: $5000 \mathrm{~V}$ (positive)/-4500 V (negative), ion source gas 1, N2: $50 \mathrm{psi}$, ion source gas 2, N2: $50 \mathrm{psi}$, auxiliary gas heating temperature (temperature/TEM): $550^{\circ} \mathrm{C}$, curtain gas/CUR: 35 psi, parent ion mass scanning range: $\mathrm{m} / \mathrm{z} 60 \sim 1500$, declustering potential/DP: $80 \mathrm{~V}$, and collision energy/CE: $10 \mathrm{eV}$. The ion scan range is $\mathrm{m} / \mathrm{z} 40 \sim 1000$; the decluster potential (DP) is $80 \mathrm{~V}$, and the collision voltage (collision energy/CE) is $35 \mathrm{eV}$. Collision energy spread (CES): 15; ion release delay (IRD): 30; ion release width (IRW): 15. 


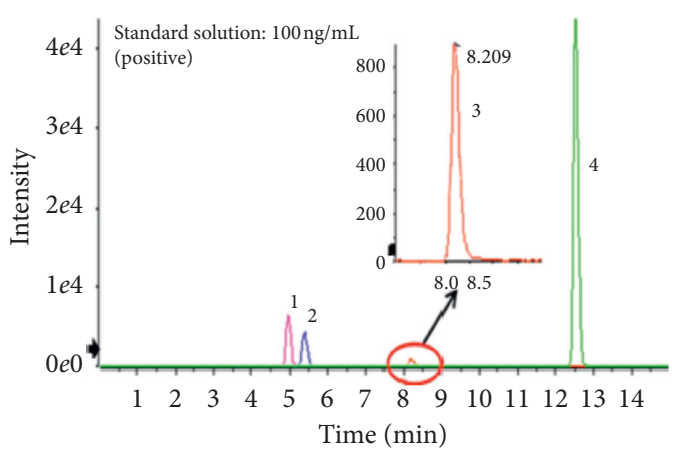

(a)

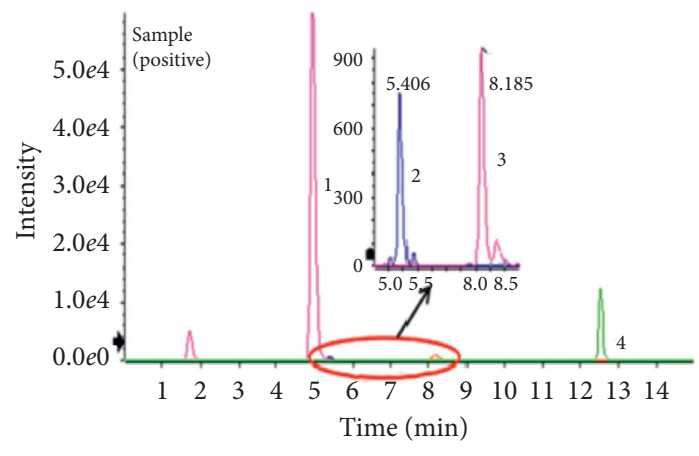

(c)

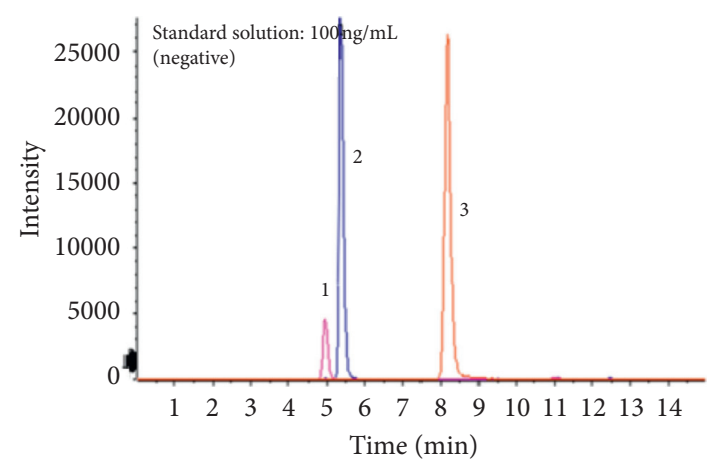

(b)

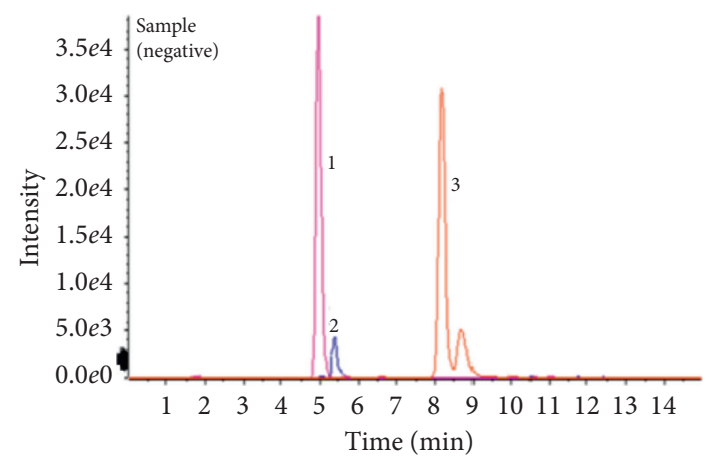

(d)

FIGURe 2: Mass spectrometry for active ingredients. XIC (extracted ion current) in positive mode (a, c) and negative mode (b, d). (a, c) Compound standard. (b, d) QFXBF extract. 1: amygdalin $\left(\mathrm{C}_{20} \mathrm{H}_{27} \mathrm{NO}_{11}\right)$, 2: tectorigenin $\left(\mathrm{C}_{16} \mathrm{H}_{12} \mathrm{O}_{6}\right)$, 3: glycyrrhizic acid $\left(\mathrm{C}_{42} \mathrm{H}_{62} \mathrm{O}_{16}\right)$, and 4: osthole $\left(\mathrm{C}_{15} \mathrm{H}_{16} \mathrm{O}_{3}\right)$.

for 7 days after 14 days of OVA intraperitoneal injection [16]. From the $7^{\text {th }}$ day after sensitization, the guinea pigs were continuously instilled with OVA by nebulization ( $10 \mu \mathrm{l}$ of $1 \mathrm{mg} / \mathrm{ml}$ per guinea pig) once a day. Starting on the $15^{\text {th }}$ day, different groups (OVA only, DXM, high QFXBF, medium QFXBF, and low QFXBF groups) were allocated separately in hermetic air containers (self-made transparent plastic boxes: $50 \times 30 \times 20 \mathrm{~cm}$ ) in the presence of $2 \%$ aerosolized inhalation OVA. The control group received physiological salt solution instead of OVA solution inhalation. The inhalation flow was $3 \mathrm{ml} / \mathrm{min}$ and administered for $20 \mathrm{~min}$ each time. We observed the animals' asthma response, including tremor, bristles, snatching, coughing, sneezing, abdominal breathing, shortness of breath, neck extension, breast reduction, head nodding, high cramping, tumbling, and exhalation, to ensure that the model is successfully established. After the appearance of the above symptoms, two guinea pigs were randomly selected from the control and OVA only groups to perform the pathological analysis. Compared to normal animals, only the OVA group had the following symptoms: bronchial mucosa and submucosa infiltrated massive eosinophilic granulocytes, bronchial wall thickening, smooth muscle hyperplasia, lumen stenosis, mild hyperemia, and edema, confirming the success of modeling.

2.5. Treatments. Intragastric administration was performed $1 \mathrm{~h}$ prior to asthma inducement; the respective doses of high
QFXBF, medium QFXBF, low QFXBF, and DXM groups were $20.7 \mathrm{~g} / \mathrm{kg}, 10.35 \mathrm{~g} / \mathrm{kg}, 5.2 \mathrm{~g} / \mathrm{kg}$, and $0.5 \mathrm{mg} / \mathrm{kg}$. The control group was injected with the same volume of sterile saline for 14 consecutive days.

2.6. Necropsy and Sample Preparation. Half of the guinea pigs were evaluated in histopathological analysis, while the rest were evaluated for gene expression. For blood sample collection, animal surgeries were performed using midline laparotomy after the guinea pigs were anaesthetized by intraperitoneal injection of $1.5 \%$ pentobarbital $(50 \mathrm{mg} / \mathrm{kg})$. The animals were then sacrificed by exsanguination from the abdominal aorta.

2.7. Bronchial Wall Thickness. Bronchial tissue sections were submitted to Masson's trichrome staining, and bronchial wall thickness was measured using a DA2000 digital medical image analysis system. Bronchial basement membrane perimeter (Pbm), mucosal epithelial layer area (WAmuc), smooth muscle area (WAm), and the area of the tracheal inner wall (WAi) were measured and normalized to $\mathrm{Pbm}$. The WAtc/Pbm, WAm/Pbm, and WAi/Pbm $\left(\mu \mathrm{m}^{2} / \mu \mathrm{m}\right)$ ratios represented the thickness of the wall for the respective bronchial layers, indicating the degree of airway remodeling.

2.8. H\&E Staining of Left Lung Samples. After killing, left lung tissue samples were collected, fixed with $10 \%$ 
formalin and embedded in paraffin. Sections $(5 \mu \mathrm{m})$ were mounted for hematoxylin and eosin (H\&E) staining. Tissue sections were observed by light microscopy to evaluated the degree of allergic inflammation and tissue remodeling.

2.9. RT-PCR. Total RNA extraction was performed by using an RNA extraction kit (TIAN-GEN). Reverse transcription was performed by the MBI Revert Aid ${ }^{\text {TM }}$ First Strand cDNA Synthesis Kit to generate cDNA. Amplification was carried out on the ABI7500 Fast Real-Time PCR System, using the TAKARA SYBR Premix Ex Taq II (Tli RNase H Plus) Kit. The primers of c-Fos, c-Jun, JNK, ERK, and $\beta$-actin are summarized in Table 3. PCR amplification was conducted as follows: initial denaturation at $95^{\circ} \mathrm{C}$ for $30 \mathrm{~s}$ and 40 cycles of $95^{\circ} \mathrm{C}$ for $10 \mathrm{~s}$, followed by $60^{\circ} \mathrm{C}$ for $30 \mathrm{~s}$. SYBR Green fluorescence readings were taken once per cycle to monitor amplification of the template. Relative expression was analyzed using the $2^{-\Delta \Delta \mathrm{Ct}}$ method.

2.10. Western Blot. Guinea pig lung tissue samples were added to $150-250 \mu \mathrm{L}$ lysates per $20 \mathrm{mg}$ and homogenized with a glass homogenizer. After sufficient lysis, the samples were centrifuged at $14,000 \mathrm{rpm}$ for $5 \mathrm{~min}$. The amounts of protein in the supernatant were determined using a Bradford Assay strictly according to the instructions. Equal amounts of protein were separated by polyacrylamide gel electrophoresis and transferred onto PVDF membranes. Then, specific mouse antibodies were added for incubation, followed by secondary antibody incubation. Finally, the ECL system was used for development. Integral optical density (IOD) values of the resulting bands were determined by a Gel-Pro analyzer and normalized to $\beta$-actin.

2.11. ELISA. Levels of IFN- $\gamma$, TNF- $\alpha$, IL-5, and ERK $1 / 2$ in serum were assessed by ELISA. Blood samples from all animal groups were centrifuged at $2500 \mathrm{~g}$ for $10 \mathrm{~min}$ to obtain serum for ELISA. Serum levels of IL-13 and IL- 4 were detected using ELISA kits (Shanghai Boao Biotechnology Corporation, Shanghai, China) following the manufacturer's protocols.

2.12. Statistical Methods. The statistical software SPSS 22.0 was employed for data analysis. Data were expressed as mean $\pm \operatorname{SEM}(\bar{x} \pm s)$, and all parameters were tested for normality. Tests for homogeneity of variance were used for data matching the normal distribution; variance was assessed using the LSD method and compared to Tamhane's T2 test. Nonparametric tests were used in multiple independent samples; with Kruskal-Wallis test $P<0.01$, the overall distribution was not the same in each group, with statistically significant differences between groups, and data were used after conversion of the two (using LSD and SNK methods). $P<0.05$ was considered to be statistically significant.

\section{Results}

3.1. Pathological Findings in the Lung Tissue Samples from Bronchial Asthma Models. To assess the degree of airway inflammation and inflammatory cell types, $\mathrm{H} \& \mathrm{E}$ staining was performed to observe the bronchial mucosa (Figure 3). After induction by OVA and $\mathrm{Al}(\mathrm{OH})_{3}$ gelatin sensitization, the bronchial mucosa and submucosa in the model group showed massive infiltration of eosinophil granulocyte (Figure 3(b)), with disorderly arranged cells, hyperplasia of the bronchial epithelium, and clustered structure with neutrophil infiltration. This reflected typical airway inflammation, including bronchial stenosis, severe cavity epithelial cell loss, and mucosal tissue edema. In the control group, the bronchioles and alveoli of the lung tissue and blood vessels were normal, as shown in Figure 3(a). In the DXM group (Figure 3(c)), the bronchial mucosal still had edema; infiltration of eosinophils and other inflammatory cells like neutrophils was improved compared with the OVA only group, although still visible. There was a small cavity of exfoliated epithelial and inflammatory cells. The high QFXBF group (Figure 3(d)) showed a slight improvement compared to the DXM group; the degrees of bronchial mucosa congestion and edema were significantly reduced, and eosinophilic infiltration was effectively alleviated.

Compared to the DXM (Figure 3(c)) and high QFXBF (Figure 3(d)) groups, the altered bronchial mucous membrane, inflammatory cell infiltration of the bronchial mucosa and submucosa tissue, and intraluminal cell shedding were more evident in the medium (Figure 3(e)) and low (Figure 3(f)) QFXBF groups. The single direction arrow represents the altered inflammatory cell infiltration in the bronchial mucosa.

3.2. Small Airway Wall Thickness in Bronchial Asthma Models. The bronchiolar thickness of the model group increased gradually over time; the bronchiolar thickness of the DXM group also increased, but at a slower rate. There were no significant differences in airway smooth muscle thickness between the two groups at $24 \mathrm{~h}$, one week, two weeks, and four weeks after challenge as shown in Table $4(P>0.05)$, and time dependence was not strong. Compared to the model group, the airway wall thickness was slightly controlled, but the difference was not significant, and the control effect was not good enough. Meanwhile, the difference between the high dose and model groups was significant $(P<0.05)$.

3.3. Relative Expression of AP-1 (including c-Fos and c-Jun Genes), JNK, and ERK1/2 in the Lung Tissue. After sensitization, the relative gene expression levels of c-Fos, c-Jun, JNK, and ERK1/2 were analyzed. As shown in Figure 4, the RNA relative expression levels of c-Fos in the DXM and high QFXBF groups were significantly reduced compared to the OVA only group $\left({ }^{*} P<0.05, n=10\right)$. The relative gene expression levels of c-Jun in the lung tissue were different compared to the DXM $\left({ }^{*} P<0.05, n=10\right)$ and OVA only $\left({ }^{\&} P<0.05, n=10\right)$ groups. These findings indicated that 
TABLE 3: The primers of targeted gene for RT-PCR assay.

\begin{tabular}{lrr}
\hline Primer & Forward $\left(3^{\prime} \longrightarrow 5^{\prime}\right)$ & Reverse $\left(5^{\prime} \longrightarrow 3^{\prime}\right)$ \\
\hline$\beta$-Actin & GCCTTCATACATCAAGTT & AATCTTCGCCTTAATACT \\
c-Fos & CTGCCAGAATGAACTCTA & AGACCAACTTGAAGATGAGAA \\
c-Jun & CGAGTTCTGCGCTTTCAAGGT & TCCAAGTGCAGGAAGAGGAAG \\
JNK & CACAATAGCCATTCCGTGCC & GCAAACAGGTGGACCAGGAT \\
ERK & CGTTGATGCCAATGATAT & TTGAACACCAGACTTACT \\
\hline
\end{tabular}

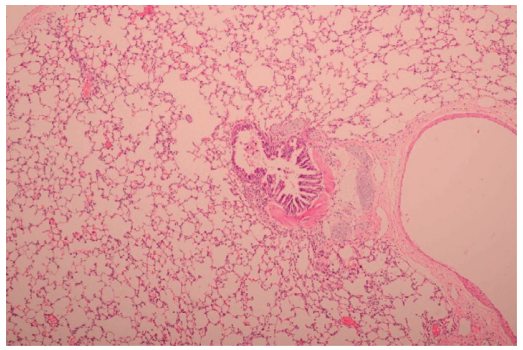

(a)

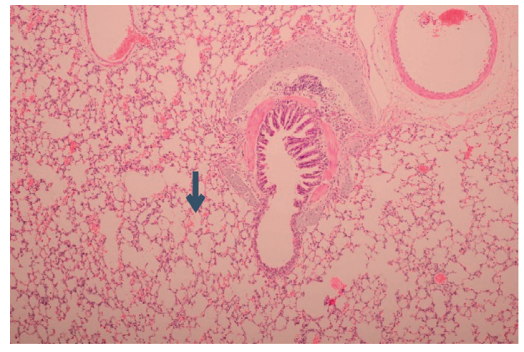

(d)

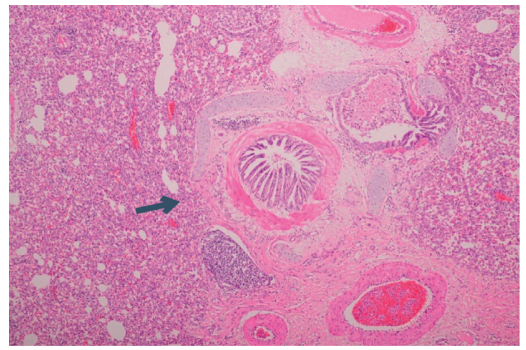

(b)

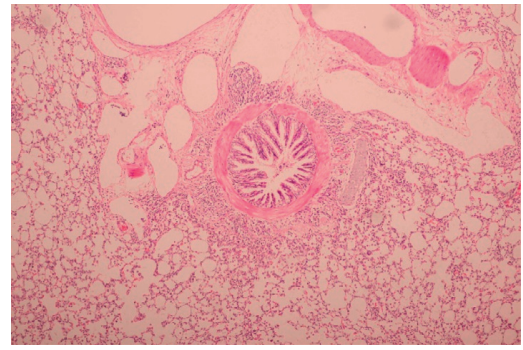

(e)

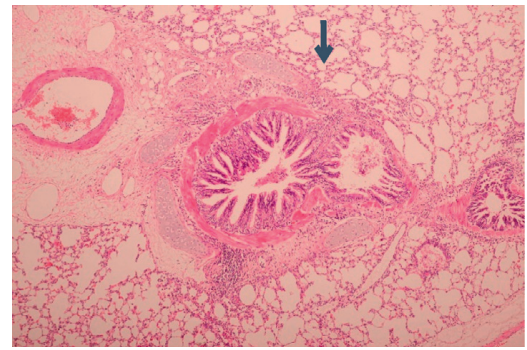

(c)

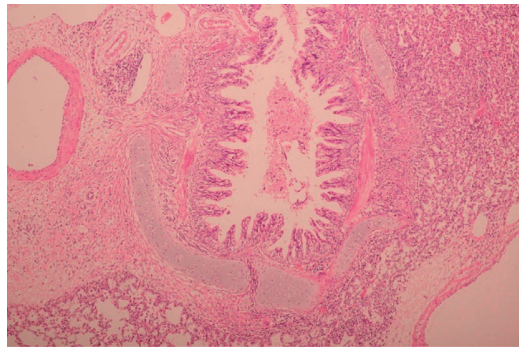

(f)

FIGURE 3: Histopathological staining of lungs from different groups: (a) control, (b) OVA only, (c) DXM, (d) high QFXBF, (e) medium QFXBF, and (f) low QFXBF groups (high QFXBF group, $20.7 \mathrm{~g} / \mathrm{kg}$; low QFXBF group, $10.35 \mathrm{~g} / \mathrm{kg}$; DXM group, $0.5 \mathrm{mg} / \mathrm{kg}$ ).

TABLE 4: Small airway smooth muscle thickness $(\bar{x} \pm \mathrm{s}, \mu \mathrm{m})$.

\begin{tabular}{lcccccc}
\hline Groups & Control group & OVA only group & DXM group & High QFXBF & Medium QFXBF & Low QFXBF \\
\hline$n$ & 10 & 10 & 10 & 10 & 10 & $7.84 \pm 1.21$ \\
$24 \mathrm{~h}$ & $7.80 \pm 1.55$ & $7.76 \pm 1.83$ & $7.98 \pm 1.46$ & $7.89 \pm 1.38$ & $7.85 \pm 1.46$ \\
$1 \mathrm{w}$ & $8.64 \pm 2.47$ & $8.44 \pm 1.98$ & $8.16 \pm 1.35$ & $8.04 \pm 1.95$ & $8.22 \pm 2.01$ & $8.38 \pm 2.20$ \\
$2 \mathrm{w}$ & $8.92 \pm 2.23$ & $11.76 \pm 2.63$ & $10.70 \pm 2.44$ & $9.96 \pm 2.27$ & $11.35 \pm 2.39$ & $11.44 \pm 2.65$ \\
$4 \mathrm{w}$ & $9.01 \pm 2.84$ & $14.42 \pm 3.87$ & $12.03 \pm 3.14$ & $10.98 \pm 2.87$ & $12.67 \pm 3.32$ & $14.06 \pm 3.19$ \\
\hline
\end{tabular}

treatment with high QFXBF could significantly reduce AP-1 expression in a concentration-dependent manner.

The relative gene expression levels of JNK showed a dramatic drop in the high QFXBF group relative to the model group $\left({ }^{*} P<0.01, n=10\right)$. Indeed all animals treated with medicinal products showed overt downregulation, but the high QFXBF group had a more pronounced improvement than the DXM group $\left({ }^{\&} P<0.05, n=10\right)$. The relative gene expression levels of ERK1/2 in the lung tissue showed markedly decreased amounts in the high QFXBF group compared to the OVA only group $\left({ }^{*} P<0.01, n=10\right)$, but no difference was observed compared to the DXM administered groups ( $\left.{ }^{\&} P>0.05, n=10\right)$.
3.4. TLR-9, c-Fos, c-Jun, and ERK1/2 Phosphorylated Protein Levels in the Lung Tissue. In a previous study [6], we found that relative expression levels of TLR-9 in the low QFXBF and high QFXBF groups were significantly higher than those in control, OVA only, and DXM groups. However, there was no overt increase in protein level in the current study compared to control values $\left({ }^{*} P>0.05, n=10\right)$.

We also evaluated c-Jun and c-Fos protein expression levels in the lung tissue (Figures 5 and 6). The high QFXBF group showed lower amounts compared with the OVA only and low QFXBF groups $\left({ }^{*} P<0.05, n=10\right)$, but no statistically significant differences were observed compared to the control, DXM, low QFXBF, and medium QFXBF groups ( ${ }^{\&} P>0.05$, 

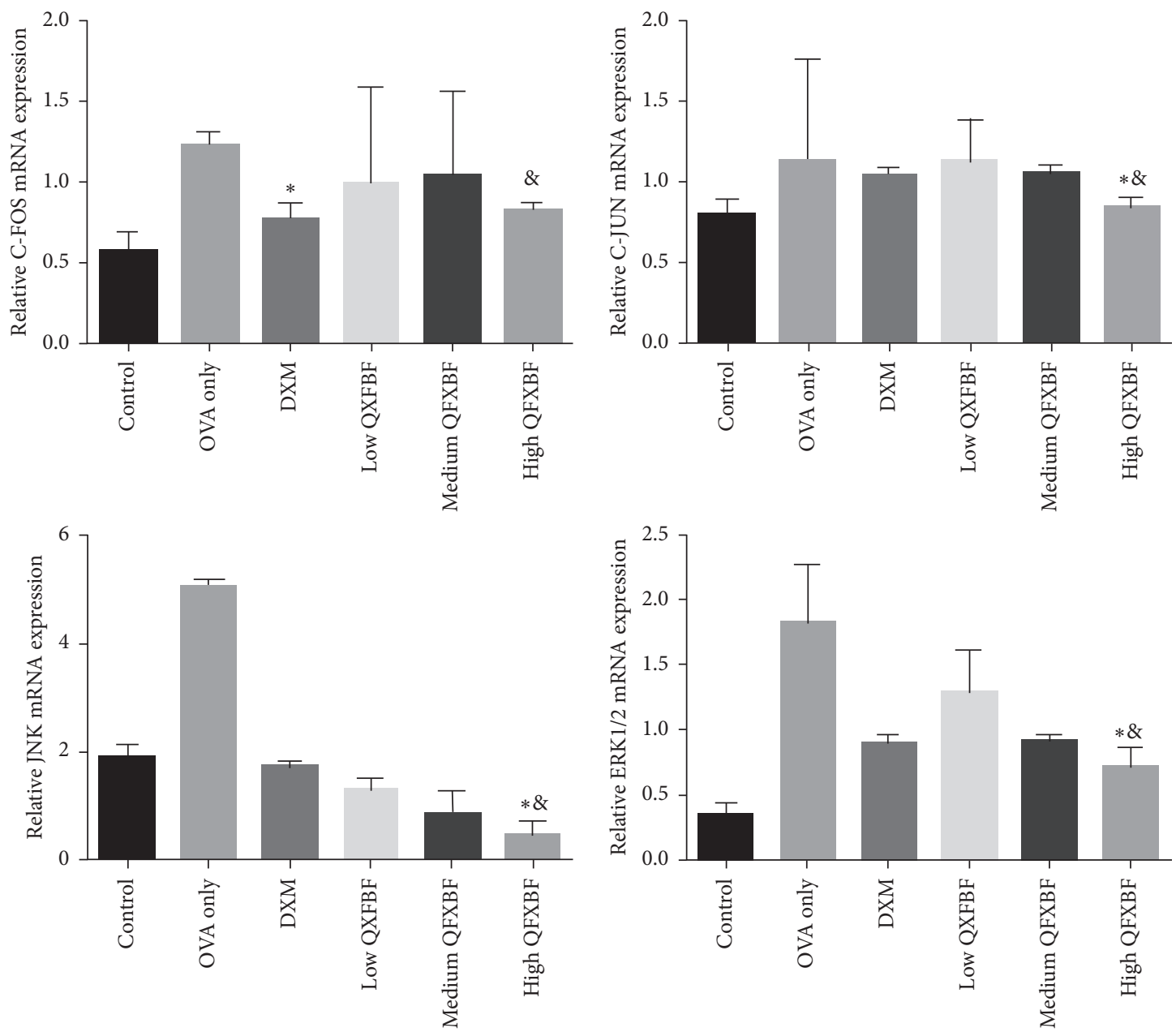

Figure 4: Relative expression levels of c-Fos, c-Jun, JNK, and ERK1/2 genes in the lung tissue.

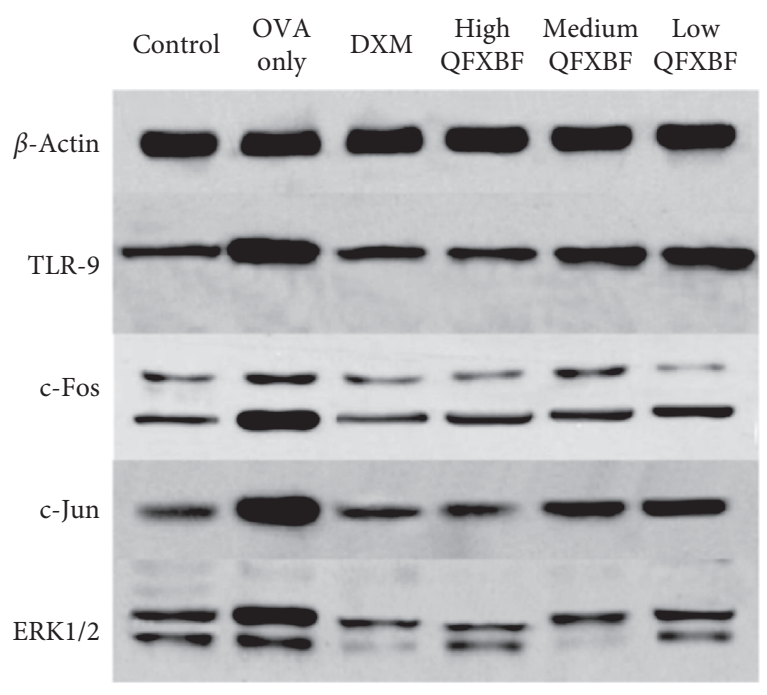

Figure 5: Protein levels of c-Fos, c-Jun, TLR-9, and ERK1/2. The protein levels were determined by immunoblot. From left to right: control, OVA only, DXM, high QFXBF, medium QFXBF, and low QFXBF groups (high QFXBF group, $20.7 \mathrm{~g} / \mathrm{kg}$; low QFXBF group, $10.35 \mathrm{~g} / \mathrm{kg}$; DXM group, $0.5 \mathrm{mg} / \mathrm{kg}$.). $n=10)$. ERK1/2 protein expression levels showed similar trends. The high QFXBF group had lower levels compared with the OVA only group ( $\left.{ }^{\&} P<0.05, n=10\right)$, but no differences were observed compared to the other treatment groups.

These data for the TLR-9 signaling pathway indicated that a high protein level of TLR-9 was not found in the tissue, while inflammatory cytokine activating proteins downstream of TLR-9 signaling were effectively downregulated; these changes allowed the control of airway inflammation.

3.5. Levels of the Th1/Th2 Inflammatory Cytokines IFN- $\gamma$, TNF- $\alpha, I L-5$, and ERK $1 / 2$ in Serum. To evaluate the levels of Th1/Th2 cytokines in serum, specific ELISA kits were used for analysis (Figure 7). Th2 cytokines, including IL-5 and TNF- $\alpha$, showed reduced serum amounts in the high QFXBF group $\left({ }^{*} P<0.01, n=10\right)$, while the Th1 cytokine IFN- $\gamma$ was significantly increased ( $\left.{ }^{\&} P<0.05, n=10\right)$, compared with the control and other treatment groups.

ERK1/2 showed a trend with the OVA only group having the highest levels; after treatment, ERK1/2 levels were controlled, and the medium and high QFXBF group showed the most apparent effects compared to the OVA only and DXM groups $\left({ }^{* \&} P<0.05, n=10\right)$. 

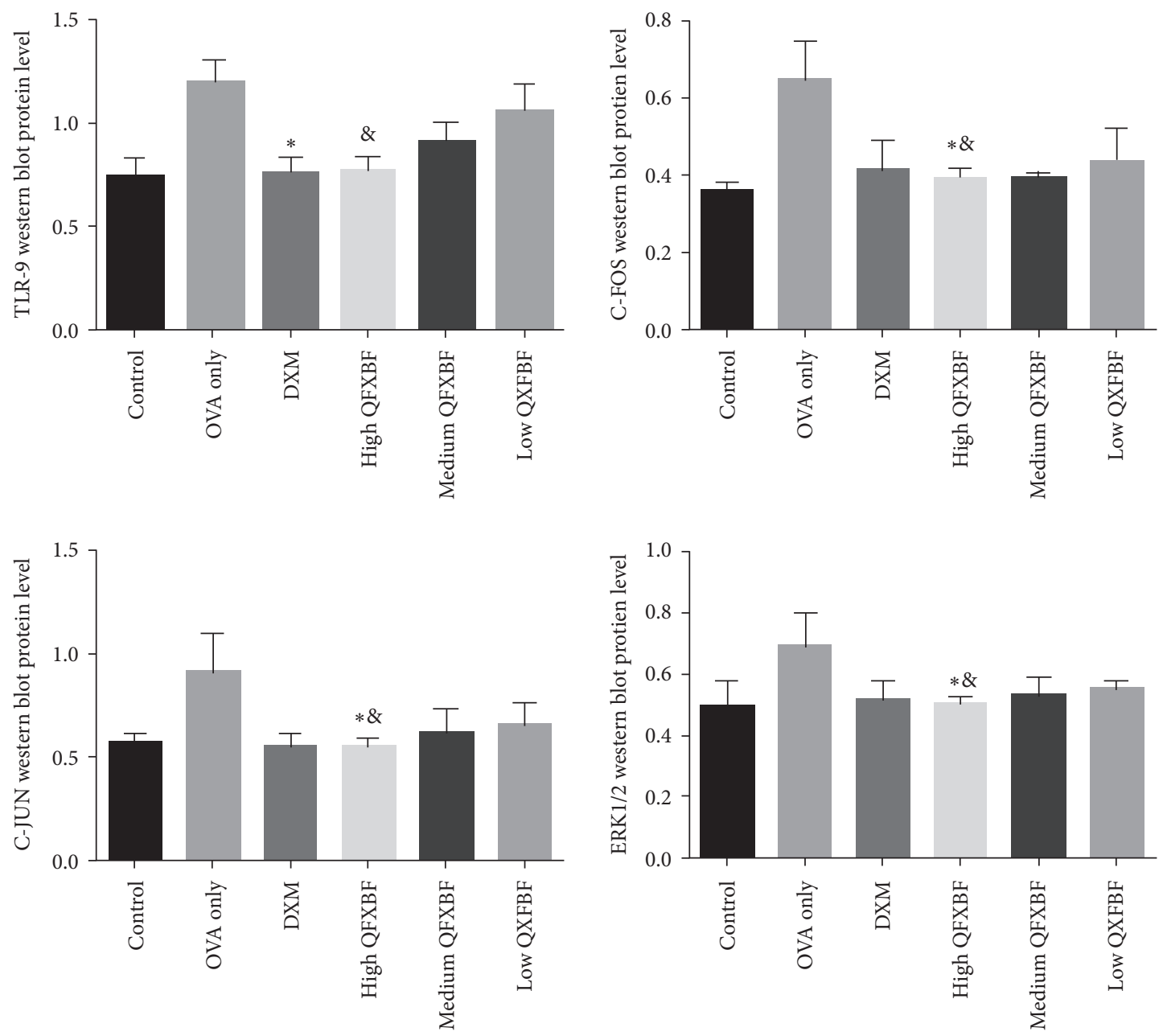

FIGURE 6: Quantitation of western blot results for target proteins in lung tissue samples from the control, OVA only, DXM, high QFXBF, medium QFXBF, and low QFXBF groups (high QFXBF group, 20.7 g/kg; low QFXBF group, 10.35 g/kg; DXM group, $0.5 \mathrm{mg} / \mathrm{kg}$.).

These findings demonstrate that the Th1/Th2 imbalance was effectively reversed after treatment with QFXBF by increasing the level of IFN- $\gamma$ and lowering the level of IL-5 level to attenuate inflammation and reconstruction in the guinea pig airway effectively. The high dose QFXBF group effectively suppresses local inflammation and regulates the immune balance compared to the DXM group.

\section{Discussion}

Airway inflammation and remodeling are two features of bronchial asthma [17], especially the latter one, which confers unique pathological structural changes, including airway epithelial damage, reticular basement membrane fibrosis or subcutaneous fibrosis, proteoglycan deposition, smooth muscle hypertrophy, goblet cell metaplasia caused by mucosal gland hyperplasia, profound changes in extracellular matrix cell position, and new blood vessel growth and proliferation. The most prominent inflammatory cell types in airway remodeling are mast cells, eosinophils, Th2 cells, and neutrophils. Specifically, Th2 cells not only participate in chronic allergic airway inflammation as previously reported but also uniquely accelerate smooth muscle cell proliferation and epithelial metaplasia, which can affect airway remodeling progression as recently shown [18].

In this study, QFXBF induced the gene expression of lung TLR-9 signaling pathways, including the subordinate JNK, ERK1/2, and other signal pathways. In order to confirm the expression of downstream inflammatory transcription factors such as TLR-9, AP-1, and ERK1/2 protein levels in the lungs, western blot was performed to assess whether QFXBF controlled the related proteins that mediate airway inflammation and remodeling. As shown above, QFXBF downregulated different proteins in the MAPK pathway, such as JNK and ERK1/2 in the TLR-9 signaling pathway, as also reported for the NF- $\kappa$ B pathway [6].

TLR-9 protein levels in the treatment groups were lower than those of the model group in this study, which does not seem to be consistent with the currently accepted theory that TLR-9 upregulation is helpful in the treatment of allergic diseases but does not negate the role of TLR-9 in the lung tissue. It is assumed that the TLR-9 gene is expressed and plays a therapeutic role in the lung tissue; however, TLR-9 may partly be degraded by lysosomal enzymes due to some reasons such as short duration of administration or Triad3A (ring-finger E3 ligase and Triad3 family member) in the ubiquitination process $[19,20]$. 

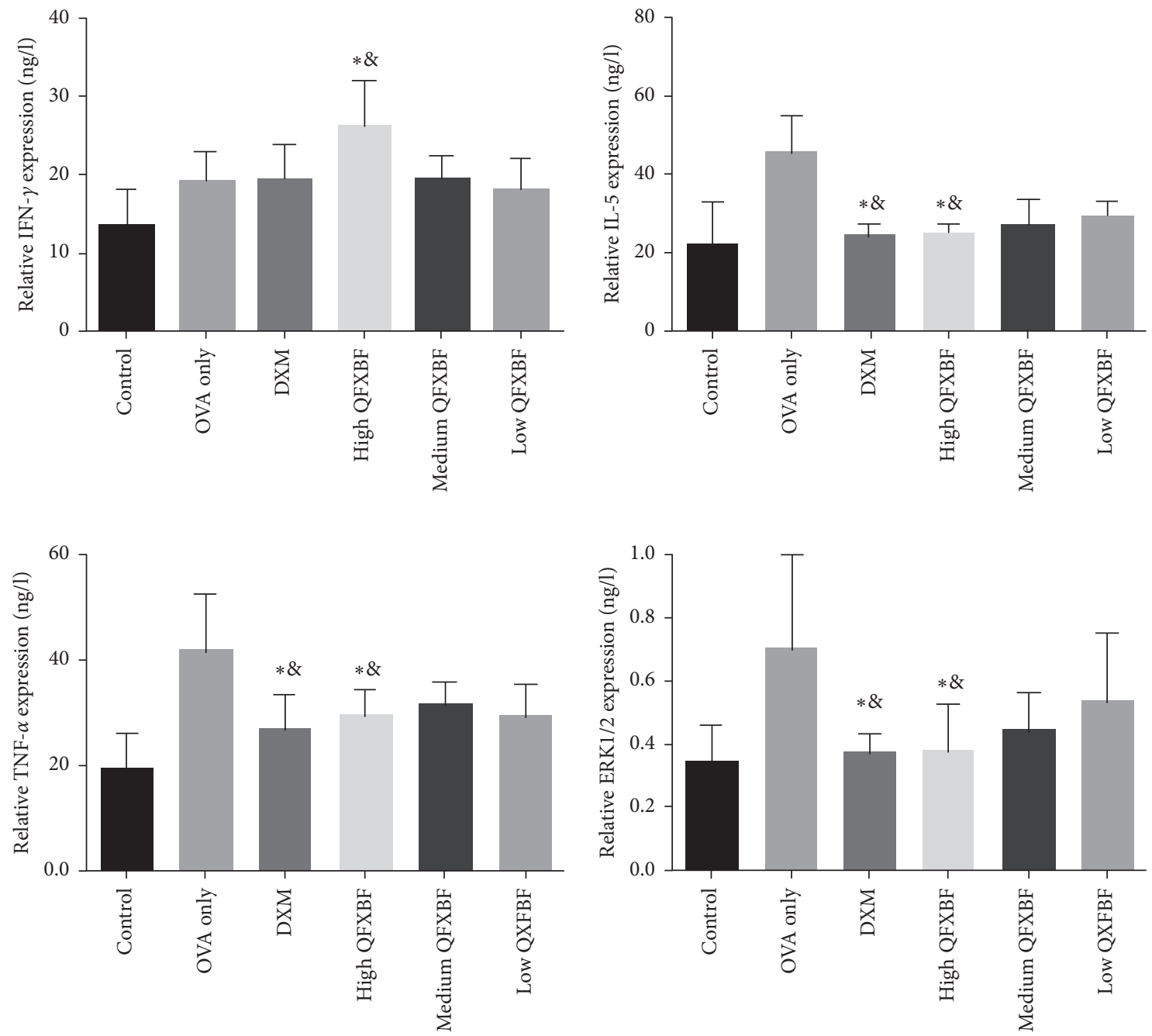

FIgURE 7: Serum levels of IFN- $\gamma$, TNF- $\alpha$, IL-5, and ERK1/2 after treatment: (a) IFN- $\gamma$; (b) TNF- $\alpha$; (c) IL-5; (d) ERK1/2.

Immune stimulation of the airway mucosa induces active T-cell-mediated immune reactions leading to tolerance or asthma. Regulation of these T-cell responses can affect major pathways throughout the airway after antigen stimulation. In this study, levels of IFN- $\gamma$ (a representative of Th1 cytokines) were increased, while the levels of Th2 cytokines IL-4 and TNF- $\alpha$ were evidently decreased. Therefore, the abnormal inflammatory response could be reversed from the Th2 type to the Th1 one. These results suggest that QFXBF might block the induction of inflammatory chemokines by inhibiting the activation of TLR-9 downstream transformation factors, restoring the Th1/Th2 balance. This is one of the most probable mechanisms of QFXBF treatment for bronchial asthma.

Unfortunately, there was no convincing evidence that QFXBF could delay airway remodeling and reduce smooth muscle thickness of the bronchial airway. In this study, highdose QFXBF was superior to the other two QFXBF doses in controlling inflammatory cytokines, suggesting a dose-dependent efficacy. Considering that guinea pigs are not that tolerant of the modeling stimulus and get easily scared, this 10-day gavage study may not be long enough to monitor the expected airway changes.
Even if positive results were achieved, it would be difficult to undoubtedly claim that ERK1/2, which is downstream of the MAPK pathway, is responsible for this effect. Traditional Chinese medicine decoction is known to have multitarget effects in improving the overall immune system. It contains surprisingly large amounts of active pharmacological ingredients that affect an extremely large number of targets and pathways. Therefore, we could not fully explain in what range and to what extent QFXBF could affect the airway remodeling process.

In summary, the present study demonstrated that following activation of TLR-9, the Th1/Th2 ratio imbalance was significantly improved after administration of QFXBF, exceptionally high dose involving the MAPK pathway with downstream cytokines. These findings indicate that QFXBF extract improves the outcome of bronchial asthma, revealing one of the possible mechanisms of this ailment, which significantly improves the treatment and management of patients with bronchial asthma and has a profound impact on the processing of medical information. 


\section{Data Availability}

The data used to support the findings of this study are available from the corresponding author upon request.

\section{Conflicts of Interest}

The authors declare that they have no potential conflicts of interest.

\section{Authors' Contributions}

Suofang Shi conceived and designed the experiments. Li Liu gave experimental guidance in the lab. Shuomiao Yin, Lingfu Wang, and Po Hu performed the experiments. Po $\mathrm{Hu}$ analyzed the data and wrote the paper. Po Hu and Suofang Shi contributed equally to this study.

\section{Acknowledgments}

This study was supported by the grants from the National Natural Science Fund Project (81774267) and the Jiangsu Provincial Science and Technology Department Provincial Natural Fund Project (no. BK20141502).

\section{References}

[1] S. Akira, S. Uematsu, and O. Takeuchi, "Pathogen recognition and innate immunity," Cell, vol. 124, no. 4, pp. 783-801, 2006.

[2] WHO, Global Surveillance, Prevention and Control of Chronic Respiratory Diseases: A Comprehensive Approach, WHO, Geneva, Switzerland, 2007.

[3] K.-M. Beeh, F. Kanniess, F. Wagner et al., "The novel TLR-9 agonist QbG10 shows clinical efficacy in persistent allergic asthma," Journal of Allergy and Clinical Immunology, vol. 131, no. 3, pp. 866-874, 2013.

[4] Z. Aryan, S. T. Holgate, D. Radzioch, and N. Rezaei, "A new era of targeting the ancient gatekeepers of the immune system: toll-like agonists in the treatment of allergic rhinitis and asthma," International Archives of Allergy and Immunology, vol. 164, no. 1, pp. 46-63, 2014

[5] J. T. Olin and M. E. Wechsler, "Asthma pathogenesis and novel drugs for treatment," British Medical Journal, vol. 349, p. g5517, 2014.

[6] K. Zhou, L. Liu, and S. Shi, "Qu Feng Xuan Bi Formula attenuates anaphylactic rhinitis-asthma symptoms via reducing EOS count and regulating T cell function in rat ARA models," Journal of Ethnopharmacology, vol. 152, no. 3, pp. 568-574, 2014.

[7] G. K. Gupta and D. K. Agrawal, "CpG oligodeoxynucleotides as TLR-9 agonists," BioDrugs, vol. 24, no. 4, pp. 225-235, 2010.

[8] K. N. Lan, W. Kolch, and B. N. Kholodenko, "When ubiquitination meets phosphorylation: a systems biology perspective of EGFR/MAPK signaling," Cell Communication \& Signaling, vol. 11, no. 1, pp. 1-15, 2013.

[9] F. Al, B. Alessandro, S. Anisha et al., "The transcriptional activator Gli2 modulates T-cell receptor signaling through attenuation of AP-1 and NF- $\kappa \mathrm{B}$ activity," Journal of Cell Science, vol. 128, no. 11, pp. 2085-2095, 2015.

[10] T. Naka, M. Fujimoto, H. Tsutsui, and A. Yoshimura, "Negative regulation of cytokine and TLR signalings by SOCS and others," Advances in Immunology, vol. 87, no. 2, pp. 61-122, 2005.

[11] B. Temizoz, E. Kuroda, K. Ohata et al., "TLR-9 and STING agonists synergistically induce innate and adaptive type-II IFN," European Journal of Immunology, vol. 45, no. 4, p. 1159, 2015.

[12] M. Kerkmann, S. Rothenfusser, V. Hornung et al., "Activation with CpG-A and CpG-B oligonucleotides reveals two distinct regulatory pathways of type I IFN synthesis in human plasmacytoid dendritic cells," The Journal of Immunology, vol. 170 , no. 9 , p. $4465,2003$.

[13] R. Takauji, S. Iho, H. Takatsuka et al., "CpG-DNA-induced IFN- $\alpha$ production involves p38 MAPK-dependent STAT1 phosphorylation in human plasmacytoid dendritic cell precursors," Journal of Leukocyte Biology, vol. 72, no. 5, p. 1011, 2002.

[14] T. Masuda, H. Tanaka, M. Komai et al., "Mast cells play a partial role in allergen-induced subepithelial fibrosis in a murine model of allergic asthma," Clinical \& Experimental Allergy, vol. 33, no. 5, pp. 705-713, 2003.

[15] W. T. Gerthoffer and C. A. Singer, "MAPK regulation of gene expression in airway smooth muscle," Journal of Respiratory Physiology and Neurobiology, vol. 137, no. 2-3, pp. 237-250, 2003.

[16] C. T. McCusker, "Use of mouse models of allergic rhinitis to study the upper and lower airway link," Current Opinion in Allergy and Clinical Immunology, vol. 4, no. 1, pp. 11-16, 2004.

[17] N. Ohkura, M. Fujimura, A. Tokuda, S. Furusho, M. Abo, and N. Katayama, "Evaluation of airway hyperresponsiveness and exhaled nitric oxide as risk factors for airway remodeling in patients with stable asthma," Allergy and Asthma Proceedings, vol. 30, no. 4, pp. 419-423, 2009.

[18] R. Berair, F. Hollins, and C. Brightling, "Airway smooth muscle hypercontractility in asthma," Journal of Allergy, vol. 2013, no. 23, pp. 1-7, 2013.

[19] T.-H. Chuang and R. J. Ulevitch, "Triad3A, an E3 ubiquitinprotein ligase regulating Toll-like receptors," Nature Immunology, vol. 5, no. 5, pp. 495-502, 2004.

[20] C. Xu, Y. Zhang, and Y. Zhang, "Regulation of autophagy by E3 ubiquitin ligase 483 Triad3A through Beclin 1 ubiquitination," Cytokine, vol. 63, no. 3, p. 311, 2013. 